\title{
El descubrimiento de nuevos fármacos, concepto umbral en farmacología.
}

\author{
Isaias Sanmartin Santos a , Francisco Revert Ros ${ }^{a}$, Celia Almela Camañas ${ }^{b}$, Ignacio Ventura \\ González ${ }^{c}$, Jesús Prieto Ruiz ${ }^{c}$, Ana Lloret Alcañizd
}

a. Departamento de Ciencias Médicas Básicas. Facultad de Veterinaria y Ciencias Experimentales. Universidad Católica de Valencia San Vicente Mártir.

b. Departamento de Producción y Salud Publica. Facultad de Veterinaria y Ciencias Experimentales. Universidad Católica San Vicente Mártir

c. Departamento de Ciencias Médicas Básicas. Facultad de Medicina. Universidad Católica de Valencia San Vicente Mártir.

d. Departamento de Fisiología. Facultad de Medicina. Universidad de Valencia.

\begin{abstract}
The so-called "threshold concepts" are present in all areas of study. Its acquisition by the student represents a milestone in the advancement of the conceptual understanding of a subject. They are often difficult to learn, but when they are overcome they generate a reconceptualization that allows progress and deepening in the subject of study. We present an activity designed to develop a key threshold concept in pharmacology, the drug discovery process. To do this, we develop a practical laboratory project that will integrate both the extraction and search for active ingredients and the simulation of some aspects of their clinical trials in the phase I.
\end{abstract}

Keywords: threshold concepts, motivation, project-based learning, pharmacology, laboratory practices.

\section{Resumen}

Los llamados "conceptos umbral" se encuentran presentes en todas las áreas de estudio. Su adquisición por el estudiante representa un hito en el avance de la comprensión conceptual de una asignatura. A menudo resultan dificiles de aprender, pero cuando son superados generan una reconceptualización que permite el progreso y profundización en la materia de estudio. Presentamos una actividad diseñada para desarrollar un concepto umbral clave en farmacología, el proceso de descubrimiento de fármacos. Para ello, desarrollamos un proyecto práctico de laboratorio que integrará tanto la extracción y búsqueda de principios activos como la simulación de algunos aspectos de sus ensayos clínicos en fase I.

Palabras clave: concepto umbral, motivación, aprendizaje basado en proyectos, farmacología, prácticas de laboratorio.

\section{Introducción}

Es habitual en los alumnos que se acercan por primera vez a la ciencia de la farmacología, mantener una serie de concepciones erróneas acerca del proceso de descubrimiento de fármacos. El alumno principiante, a menudo mantiene la creencia de que las moléculas terapéuticas que se utilizan en los medicamentos proceden de un proceso de invención dirigido, en el que uno o más científicos químico-farmaceúticos 
diseñan intencionalmente moléculas con actividad farmacológica adecuada para tratar una enfermedad. Sin embargo, el verdadero proceso de descubrimiento de fármacos es radicalmente diferente. Aunque en la actualidad el proceso de "invención" de moléculas activas mediante el diseño computacional apoyado en el conocimiento de la estructura del receptor ya es una realidad, la mayoría de moléculas de fármacos disponibles en el mercado son el fruto de un proceso de descubrimiento que podríamos llamar de "ensayo y error", y que sigue siendo el modelo más utilizado. Los laboratorios producen extractos de diversas especies vegetales y animales, que luego someten a toda una batería de pruebas de laboratorio para determinar si alguno de los extractos muestra algún tipo de actividad farmacológica interesante (antibacteriana, antitumoral, anticoagulante, etc.). Es un proceso de búsqueda al azar, casual, intentando revelar mediante diversas pruebas y test si existe en el extracto algún principio activo interesante.

Si el laboratorio detecta alguna molécula activa y demuestra que es segura y eficaz en animales de experimentación, pasa a ensayarse posteriormente en humanos en los llamados “ensayos clínicos". En una primera fase la molécula se administrará a voluntarios humanos sanos, en condiciones muy controladas y bajo rigurosa supervisión médica. Si resulta segura en humanos, es decir, si no produce efectos secundarios o reacciones adversas graves en estos voluntarios, pasa a ensayarse en una segunda fase en voluntarios humanos que padecen la enfermedad que la molécula trata de curar. Si resulta exitoso el tratamiento, se vuelve a ensayar en la llamada "fase III" en pacientes voluntarios, pero esta vez en un gran número de ellos, para lograr resultados estadísticamente significativos. Finalmente, si el ensayo en fase III en humanos es superado con éxito, el laboratorio intentará registrar o patentar la molécula: ha nacido un nuevo fármaco.

Como vemos, el procedimiento real de descubrimiento de fármacos es muy diferente a la idea preconcebida y equivocada del estudiante, que piensa que los medicamentos se desarrollan mediante un proceso de diseño químico dirigido, en el que el químico-farmaceútico desarrolla "sobre el papel" una sustancia química apropiada para el tratamiento de una enfermedad. Se trata de un "concepto umbral" (Biggs, 2011), que dificulta el aprendizaje, pero del que tras su superación desarrolla en el estudiante una nueva perspectiva que permite el progreso en la materia en la que trata de formarse.

Los conceptos umbral son aquellos que, cuando se entienden correctamente, provocan cambios en la perspectiva del alumno sobre la materia que estudia y, consecuentemente, cambios en el nivel de comprensión conceptual y el ulterior aprendizaje de la asignatura (Meyer, 2006).

En el diseño de los programas de estudio, se debe prestar una especial atención a estos conceptos, de manera que los estudiantes logren mediante actividades específicamente diseñadas su adquisición y aprendizaje. Dado que estos conceptos son clave para entender correctamente una materia, debemos asegurarnos de que los alumnos sean adecuadamente instruidos en los "conceptos umbral" para lograr una correcta asimilación. Los aprendizajes superficiales y la simple repetición de frases y definiciones, tan habituales, deben ser completamente evitados.

El proyecto constituye una mejora del proceso de aprendizaje en la asignatura conducente a la adquisición de un "concepto umbral" esencial: cómo se trabaja en la investigación farmacéutica para descubrir nuevos fármacos. Proporciona también una experiencia que acerca al estudiante a la actividad profesional real en la investigación de nuevos fármacos. Adicionalmente, enseña las técnicas o ensayos de laboratorio reales que se utilizan para descubrir nuevos principios activos. Y supone una experiencia de aprendizaje activo en los "ensayos clínicos", de los que usualmente no se proporciona más formación que su exposición a nivel teórico en las distintas materias universitarias que los tratan. Los contextos de aprendizaje en los que se presentan las actividades de enseñanza-aprendizaje son un aspecto clave en la motivación de las actividades para los estudiantes. Para que una tarea sea vista como "motivadora", debe ser vista como útil para su formación, además de asequible o "realizable" (Pintrich, 2002). La motivación es muy importante en la generación de contextos o climas de aprendizaje adecuados. El proyecto es en sí mismo muy vistoso, 
ilusionante y fácil de realizar para los estudiantes, ya que la selección de técnicas que se utilizan ha sido seleccionada con ese propósito. Su perspectiva o proyección "profesional" le dota de un atractivo adicional que genera el deseado contexto motivador en los alumnos que abordan el proyecto.

La actividad se realiza con los alumnos organizados en pequeños grupos, mediante aprendizaje cooperativo, y se enmarca dentro del denominado "aprendizaje basado en proyectos" (Markham, 2003), que se desarrolla como una serie de prácticas de laboratorio realizadas en días sucesivos.

La asignatura escogida para la realización del proyecto es Farmacología para Biotecnología, que se imparte durante el cuarto curso del Grado en Biotecnología de la Universidad Católica de Valencia San Vicente Mártir.

\section{Objetivos}

Los objetivos generales que persigue el proyecto son:

1. Desarrollar el concepto umbral acerca del proceso de descubrimiento de nuevos fármacos mediante una simulación completa del proceso, desde la extracción de los principios activos hasta los ensayos clínicos

2. Introducir los métodos básicos y exponer a los alumnos a una variedad de técnicas del laboratorio de investigación en farmacología

\section{Desarrollo de la innovación}

\subsection{Búsqueda de principios activos}

Se solicitó a los estudiantes que realizaran una serie de ensayos de búsqueda de principios activos (los que se recogen en la tabla 1) sobre los materiales biológicos entregados: diente de león o achicoria amarga (Taraxacum officinale), tomillo (Thymus vulgaris), espliego (Lavandula angustifolia) y cúrcuma (Curcuma longa).

Tabla 1. Test realizados con los extractos

\begin{tabular}{ccc}
\hline Tipo de ensayo & $\begin{array}{c}\text { Actividad } \\
\text { farmacológica }\end{array}$ & Descripción \\
\hline $\begin{array}{c}\text { Citotoxicidad en } \\
\text { células Hela }\end{array}$ & Antitumoral & $\begin{array}{c}\text { Efecto citotóxico del } \\
\text { extracto sobre células } \\
\text { tumorales }\end{array}$ \\
Antibiograma & Antibiótico & $\begin{array}{c}\text { Inhibición del } \\
\text { crecimiento bacteriano } \\
\text { en placa }\end{array}$ \\
$\begin{array}{c}\text { Ensayo de } \\
\text { eliminación de }\end{array}$ & Antioxidante & $\begin{array}{c}\text { Determinación de la } \\
\text { capacidad antioxidante } \\
\text { radicales libres con }\end{array}$ \\
DPPH & & reducción de DPPH \\
& & \\
\hline
\end{tabular}

En primer lugar, los estudiantes efectuaron una extracción etanólica de los materiales. Los estudiantes emplearon dos procedimientos para la extracción:

a) Sistema Soxhlet.

(c)) BY-NC-ND 2021, Universitat Politècnica de València

Congreso In-Red (2021) 
b) Agitación con calor $\left(75^{\circ} \mathrm{C}\right)$
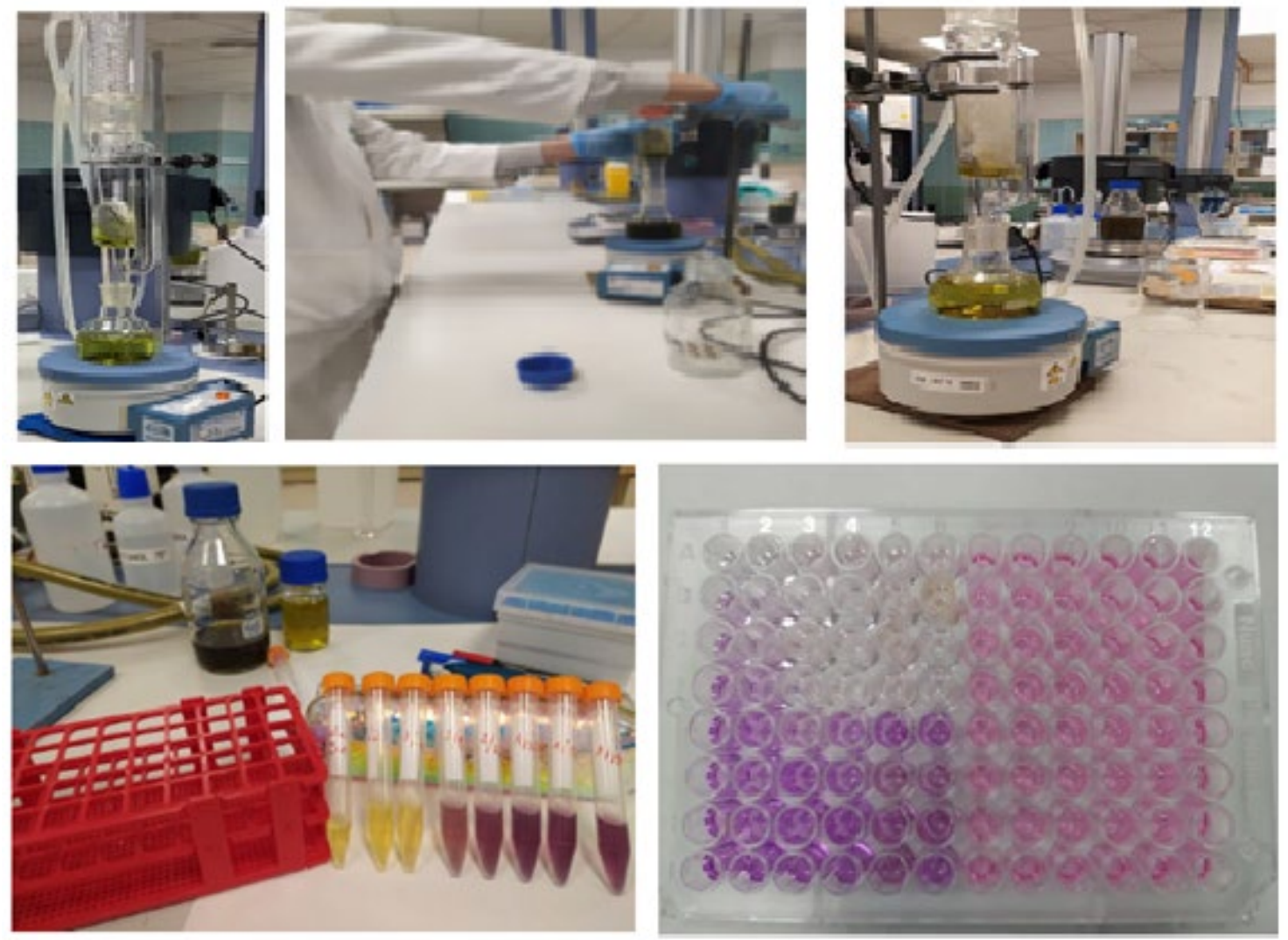

Fig. 1. Arriba: Extracción de principios activos mediante el sistema Soxhlet. Abajo, izquierda: Resultado de un ensayo con DPPH usando distintas diluciones de un extracto etanólico. Abajo, derecha: Resultado de un ensayo MTT usando células tumorales HeLa $y$ distintas diluciones de extractos resuspendidos en DMSO.

Acabada la extracción, parte de los extractos se secaron en campana de extracción, y parte se conservaron en etanol para ensayos de actividad antioxidante. La actividad antioxidante de los extractos fue determinada por los estudiantes mediante incubación con 2,2-Diphenyl-1-picrylhydrazyl (DPPH), representando la absorbancia a $517 \mathrm{~nm}$ obtenida en el ensayo, y ajustando una curva sigmoidea mediante el programa GraphPad Prism, con lo que calcularon la cantidad de material de partida con el que se obtenía la mitad del efecto máximo (ED50).
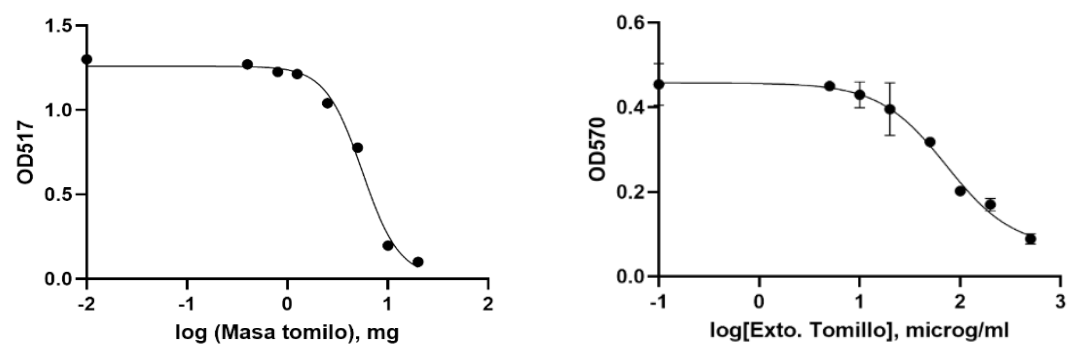

Fig. 2. Izquierda: curva dosis-respuesta obtenida con distintas diluciones de un extracto en un ensayo de capacidad antioxidante. Derecha: curva concentración-respuesta obtenida con extracto de tomillo en un ensayo MTT.

Los extractos secos fueron resuspendidos en dimetil sulfóxido (DMSO) a $100 \mathrm{mg} / \mathrm{ml}$, tras lo que se evaluó la actividad citotóxica de los mismos mediante el ensayo MTT. Células HeLa de cáncer de cérvix fueron sembradas en placas de 96 pocillos a razón de 2500 células por pocillo. La representación de los valores de 
absorbancia respecto al logaritmo de la concentración de extracto permitió obtener una curva sigmoidea y el valor de la EC50 o concentración de extracto a la que se consigue la mitad del efecto máximo. El extracto de diente de León debe mostrar actividad antitumoral (Ovadje, 2016).

En la figura 2 se muestran a modo de ejemplo los resultados obtenidos en estas experiencias con el extracto etanólico de tomillo.

\subsection{Ensayo clínico en fase I de una de las moléculas activas encontradas}

La simulación del proceso de ensayo clínico en fase I obviamente no puede realizarse con personas reales, se realizará en cambio en un modelo hidráulico denominado "el hombre de agua". El hombre de agua es un modelo monocompartimental de eliminación de fármacos (Burchum, 2019) que simula la administración del medicamento a un paciente. Se toman muestras de "sangre" y "orina" y se calculan parámetros farmacocinéticos como la semivida o la constante de eliminación, a partir de la medida de estas muestras.

En esta práctica, se dispone un modelo sencillo del organismo humano, basado en un matraz kitasatos que se drena constantemente sobre un vaso de precipitados mediante un tubo de goma conectado al matraz; este drenaje simula la eliminación renal ("orina"). El nivel de líquido se mantiene constante reponiéndolo en continuo desde un depósito de agua, que se hace gotear sobre el matraz mediante un tubo de goma. Un agitador magnético mantiene el sistema en continua agitación. Un esquema del montaje sería el que se muestra en la figura 3. El "principio activo" (extracto vegetal coloreado) se inyecta con una jeringa sobre el líquido del matraz, que representa la "sangre"
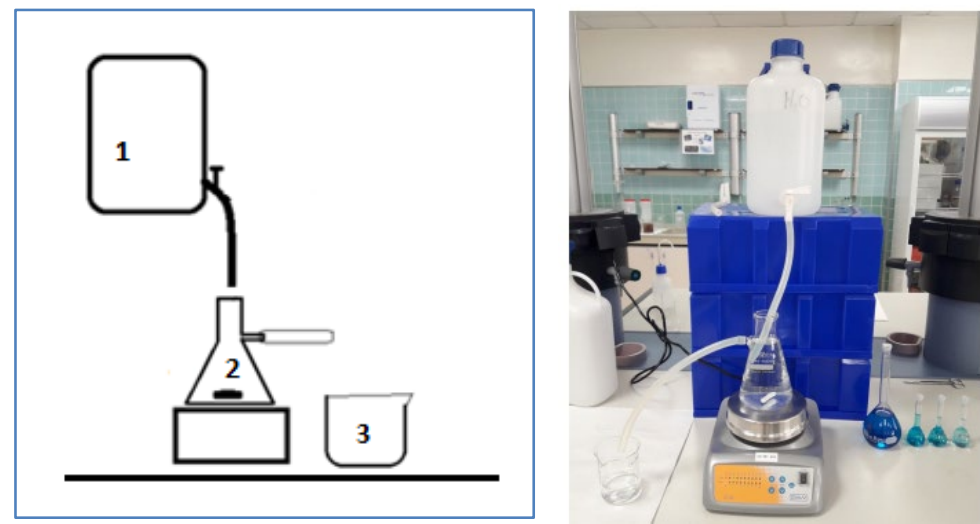

Fig. 3 A la izquierda, esquema del montaje "el hombre de agua". El depósito de agua (1) gotea sobre el matraz kitasatos que representa el compartimento acuoso central (2) que a su vez se drena sobre un vaso de precipitados (3) mediante un tubo de goma, representando la orina del paciente. A la derecha, el montaje ya terminado

El modelo está basado en la asunción de que el fármaco se distribuye únicamente por los fluidos acuosos extracelulares del organismo (modelo monocompartimental). A partir del modelo, obtendremos datos experimentales de la evolución de la concentración del "principio activo" (extracto vegetal coloreado) con el tiempo, en muestras de "plasma" y "orina", que utilizaremos para calcular los diferentes parámetros farmacocinéticos de nuestro fármaco. Estudiaremos el caso de la administración de un bolus IV a dosis única. Los estudiantes deben tomar, a los tiempos establecidos desde la administración del "fármaco", una muestra de:

a. La "sangre" del paciente, con la jeringa

b. Una muestra de "orina" del paciente, con el frasco de muestras de orina 

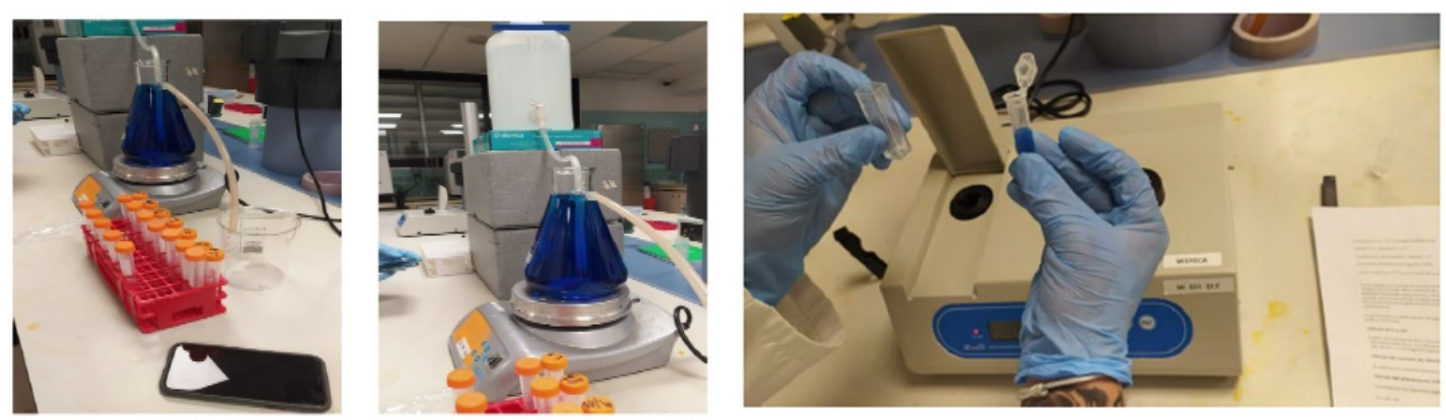

Fig. 4 A la izquierda y centro, el dispositivo "el hombre de agua", en funcionamiento; a la derecha, medida espectrofotométrica de las muestras de "sangre" $y$ "orina".

Seguidamente, determinarán con el espectrofotómetro la absorbancia del "fármaco" en las muestras. Para elaborar después con estos datos los gráficos de concentración plasmática $v s$ tiempo, y determinar a partir de ellos los parámetros farmacocinéticos requeridos. El procedimiento es similar, aunque adaptado a unas prácticas de laboratorio, al proceso real de determinación de parámetros farmacocinéticos en pacientes voluntarios en un ensayo clínico en fase I.

\section{Resultados}

Los estudiantes han acogido con agrado aprender las técnicas de ensayo de principios activos en el marco de un contexto similar al de la actividad profesional real, en lugar de un aprendizaje individual y compartimentado de cada experiencia. Como valoración subjetiva, podemos afirmar que el proyecto ha sido muy motivador y ha resultado eficaz en conseguir los objetivos que se pretendían.

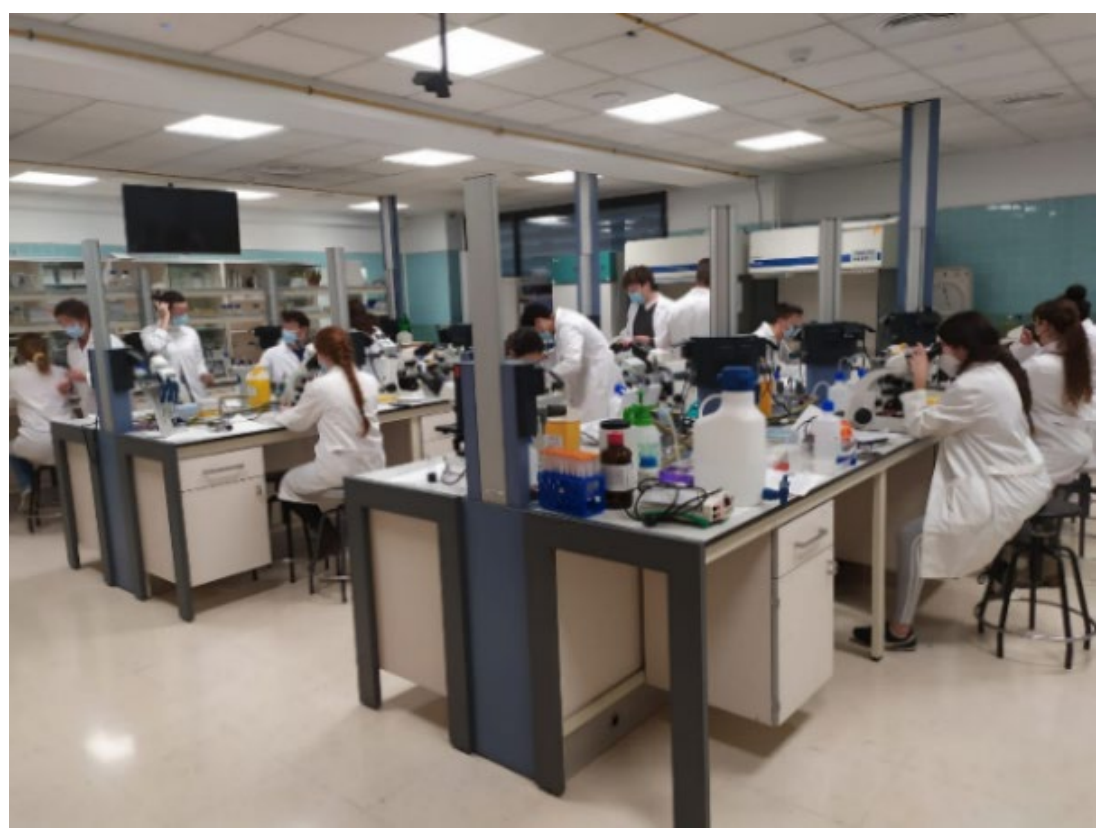

Fig. 5 Laboratorio de prácticas, durante la realización del proyecto. 
Sin embargo, para evaluarlo de manera más objetiva, hemos diseñado la siguiente encuesta. Fue presentada a los estudiantes hacia el final del curso, y los resultados se muestran en la figura 6.

Las opciones de respuesta del cuestionario están basadas en una escala Likert:

$$
\begin{aligned}
& \text { - A: totalmente en desacuerdo - B: en desacuerdo } \\
& \text { - C: ni de acuerdo ni en desacuerdo - D: de acuerdo } \\
& \text { - E: totalmente de acuerdo }
\end{aligned}
$$

\begin{tabular}{|c|c|c|}
\hline $\begin{array}{l}\text { Preg. 1: El proyecto de prácticas me ha parecido } \\
\text { interesante y atractivo en su presentación de la } \\
\text { obtención de fármacos a partir de fuentes } \\
\text { naturales. }\end{array}$ & $\begin{array}{l}\text { Preg. 2: Antes de realizar las prácticas, pensaba que } \\
\text { moléculas de los fármacos se diseñaban por los } \\
\text { químicos farmacéuticos en un proceso de invención } \\
\text { dirigido }\end{array}$ & $\begin{array}{l}\text { Preg. 3: Estas prácticas han cambiado mi } \\
\text { perspectiva acerca de cómo se obtienen los } \\
\text { fármacos }\end{array}$ \\
\hline $\begin{array}{l}\text { Preg. 4: Los protocolos del proyecto me han } \\
\text { parecido accesibles como estudiante }\end{array}$ & $\begin{array}{l}\text { Preg. 5: He asimilado con más interés los ensayos } \\
\text { integrados en el proyecto, que si me los hubiesen } \\
\text { explicado como contenidos independientes }\end{array}$ & $\begin{array}{c}\text { Preg. 6: Me gustaría que hubiesen otros } \\
\text { proyectos similares en otras prácticas de otros } \\
\text { cursos del Grado }\end{array}$ \\
\hline
\end{tabular}

Fig. 6 Resultados del cuestionario final realizada a los alumnos para cada una de las pregunta de la encuesta.

\section{Conclusiones}

El proyecto consiste en una simulación completa de todo el proceso de descubrimiento de fármacos, incluyendo la fase de ensayo clínico. No existe en nuestro conocimiento, publicada una práctica universitaria que abarque el proceso completo en toda su extensión. Para ello, proporcionamos a los estudiantes varias plantas medicinales y el material necesario para descubrir en ellas potenciales principios activos, como por ejemplo antitumorales capaces de eliminar las células de un tumor.

La totalidad de los estudiantes han respondido en la encuesta que el proyecto les ha resultado atractivo y les gustaría que hubiesen otros similares a lo largo del Grado. Además, han encontrado los procedimientos experimentales accesibles, y los han seguido con interés al contextualizarse dentro del marco integrador del proyecto. La mayoría opinan que, como se pretendía en los objetivos de aprendizaje, sus ideas sobre el proceso de descubrimiento de los fármacos han cambiado.

El hecho de que el alumno realice por sí mismo la simulación completa de todo el proceso, adicionado de la motivación e interés que generan el "descubrir" principios activos con una actividad antitumoral real y otras actividades farmacológicas, cambian por completo su perspectiva sobre el descubrimiento de fármacos. La actividad está diseñada para establecer este importante "concepto umbral" del aprendizaje de la farmacología, proporcionando una aproximación realista acerca de cómo es la actividad profesional real en la investigación de principios activos farmacológicos. 
El descubrimiento de nuevos fármacos, concepto umbral en farmacología.

\section{Referencias}

BIGGS J., TANG C. (2011) Teaching for Quality Learning at University. Society for Research Into Higher Education: London, Open University Press; 4 edition.

BURCHUM J., ROSENTHAL L. (2019). Lehne's Pharmacology for Nursing Care, Elsevier, 10th Edition.

MARKHAM, T. (2003). Project Based Learning, a guide to Standard-focused project based learning for middle and high school teachers. USA: Buck Institute for Education.

MEYER J., LAND R. (2006) Overcoming Barriers to Student Understanding. London: Routledge.

OVADJE P. et al. "Dandelion root extract affects colorectal cancer proliferation and survival through the activation of multiple death signalling pathways" Oncotarget. 2016;7(45):73080-73100. doi:10.18632/oncotarget.11485

PINTRICH, P.R., SCHUNK, D.H. (2002) Motivation in Education: Theory, Research, and Applications. Upper Saddle River, NJ: Merrill-Prentice Hall.

HANGUN-BALKIR Y., MCKENNEY M. (2012) "Determination of antioxidant activities of berries and resveratrol", Green Chemistry Letters and Reviews, 5:2, 147-153. 